TITLE:

THE EXISTENCE AND THE

CONTINUATION OF HOLOMORPHIC SOLUTIONS FOR CONVOLUTION EQUATIONS IN A HALF-SPACE IN $\$ C^{\wedge} n \$$

$\operatorname{AUTHOR}(S)$ :

OKADA, JUN-ICHI

CITATION:

OKADA, JUN-ICHI. THE EXISTENCE AND THE CONTINUATION OF HOLOMORPHIC SOLUTIONS FOR CONVOLUTION EQUATIONS IN A HALF-SPACE IN \$C^n\$. 数理解析研究所講究録 1997, 983: 70-75

ISSUE DATE:

1997-03

URL:

http://hdl.handle.net/2433/60941

RIGHT: 


\title{
THE EXISTENCE AND THE CONTINUATION OF HOLOMORPHIC SOLUTIONS FOR CONVOLUTION EQUATIONS IN A HALF-SPACE IN $\mathrm{C}^{n}$
}

\author{
JUN-ICHI OKADA \\ Department of Mathematics and Informatics \\ Institute of Science and Technology \\ Chiba University \\ 1-33 Yayoi-cho, Inage-ku, Chiba, 263 Japan \\ E-mail : mokada@math.s.chiba-u.ac.jp
}

\begin{abstract}
We study holomorphic solutions for convolution equations (E) $T * f=g$ in a half-space in $\mathbf{C}^{n}$. Under a natural condition (the condition (S)), we will prove the existence of solutions of $(\mathrm{E})$ and the analytic continuation of homogeneous equation ( $\left.\mathrm{E}^{\prime}\right) T * f=0$.
\end{abstract}

\section{INTRODUCTION}

Let $\Omega$ be a convex domain and let $K$ be a compact convex set in $\mathrm{C}^{n}$. We denote by $\mathcal{O}(\Omega)$ the space of holomorphic functions on $\Omega$ provided with the topology of uniform convergence on compact subsets of $\Omega$, and by $\mathcal{O}(K)$ the space of germs of functions holomorphic on 
$K$, endowed with the usual topology of the inductive limit. Then each nonzero analytic functional $T \in \mathcal{O}^{\prime}\left(\mathbf{C}^{n}\right)$ carried by $K$ (or equivalently, $T \in \mathcal{O}^{\prime}(K)$ ) defines a continuous linear convolution operator

$$
T *: \mathcal{O}(\Omega+K) \longrightarrow \mathcal{O}(\Omega)
$$

which is given by

$$
(T * f)(z)=T_{\zeta}(f(z+\zeta)), z \in \Omega .
$$

If $K=\{0\}$, the convolution operator $T *$ is a linear partial differential operator of infinite order with constant coefficients on $\mathcal{O}(\Omega)$. The convolution equation has been historically studied by many authors, especially the equation in the category of holomorphic functions defined on a complex domain. For example, using the notion of an entire function of completely regular growth on a fixed ray, Morzhakov [1] established sufficient condition for $T *$ to be surjective in the general case, and gave a criterion for the solvability for three classes of domains: smooth domains, products of smooth planar domains, and domains whose boundaries consist of smooth points and vertices. On the other hand, under the condition (S) due to Kawai [2], Ishimura - Y. Okada [3] studied the existence and the continuation problem of holomorphic solutions for convolution equations of hyperfunction kernels in the tube domain. In [4], Ishimura and the author proved that the property of completely regular growth is equivalent to the condition $(\mathrm{S})$ for entire functions, in more general case, i.e. for sub-harmonic functions.

In this paper, we consider the convolution equation in the case where $\Omega$ is a half-space, and under the condition $(\mathrm{S})_{\zeta_{0}}$, we will prove the existence of solutions of $(\mathrm{E})$ and the analytic continuation of homogeneous equation $\left(\mathrm{E}^{\prime}\right)$.

Most of results is based on the paper [3], and refer to it for the details of proofs.

\section{PRELIMINARIES}

Let

$$
|z|^{2}=z_{1} \overline{z_{1}}+\cdots+z_{n} \overline{z_{n}}, \quad\langle z, w\rangle=z_{1} \overline{w_{1}}+\cdots+z_{n} \overline{w_{n}}
$$

for

$$
z=\left(z_{1}, \cdots, z_{n}\right), w=\left(w_{1}, \cdots, w_{n}\right) \in \mathbf{C}^{n} .
$$

For $\zeta_{0} \in \mathbf{C}^{n}$ and $\left|\zeta_{0}\right|=1$, we put

$$
\Omega=\left\{\zeta \in \mathbf{C}^{n} \mid \operatorname{Re}\left\langle\zeta_{0}, \zeta\right\rangle<0\right\}
$$


and take a compact convex set $K$ as $K \subset \Omega$, i.e. $\Omega+K=\Omega$. As it is well-known, the properties of convolution operator are reflected in the properties of the Fourier-Borel transform of $T$, namely

$$
\widehat{T}(\zeta)=T_{z}(\exp \langle z, \zeta\rangle)
$$

which is an entire function of exponential type satisfying the following estimate:

Theorem 2.1.(Polyà-Ehrenpreis-Martineau) If $T \in \mathcal{O}^{\prime}\left(\mathbf{C}^{n}\right)$ and $T$ is carried by a compact set $K \subset \mathbf{C}^{n}$, then $\widehat{T}(\zeta)$ is an entire function and for every $\varepsilon>0$, there is a constant $C_{\varepsilon}>0$ such that

$$
|\widehat{T}(\zeta)| \leq C_{\varepsilon} \exp \left(H_{K}(\zeta)+\varepsilon|\zeta|\right), \quad \zeta \in \mathbf{C}^{n}
$$

where $H_{K}(\zeta)=\sup _{z \in K} \operatorname{Re}\langle z, \zeta\rangle$ is the supporting function of $K$.

Conversely, if $K$ is a compact convex set and $f(\zeta)$ an entire function satisfying (2.1) for every $\varepsilon>0$, there exists an analytic functional $T \in \mathcal{O}^{\prime}\left(\mathbf{C}^{n}\right)$ carried by $K$ such that $\widehat{T}(\zeta)=f(\zeta)$.

In this paper, we suppose the following condition for the entire function $\widehat{T}(\zeta)$.

Definition 2.2. We say that $\widehat{T}(\zeta)$ satisfies the condition (S) to the direction $\zeta_{0}$ or simply it satisfies the condition $(\mathrm{S})_{\zeta_{0}}$ if we have

$$
\left\{\begin{array}{l}
\text { For every } \varepsilon>0, \text { there exists } N>0 \text { such that } \\
\text { for any } r \in \mathbf{R} \text { with } r>N, \\
\text { we can find } \zeta \in \mathbf{C}^{n}, \text { which satisfies } \\
\left|\zeta-\zeta_{0}\right|<\varepsilon r \\
|\widehat{T}(\zeta)| \geq \exp (-\varepsilon r) .
\end{array}\right.
$$

\section{THE EXISTENCE OF HOLOMORPHIC SOLUTIONS}

We will prove the surjectivity theorem under the condition $(\mathrm{S})_{\zeta_{0}}$.

Theorem 3.1. Let $T \in \mathcal{O}^{\prime}\left(\mathbf{C}^{n}\right)$ carried by $K$. Assume that $\widehat{T}(\zeta)$ satisfies the condition $(\mathrm{S})_{\zeta_{0}}$. Then the convolution operator

$$
T *: \mathcal{O}(\Omega+K) \longrightarrow \mathcal{O}(\Omega)
$$


is surjective.

Proof. The transpose of

$$
P=T *: \mathcal{O}(\Omega+K) \rightarrow \mathcal{O}(\Omega)
$$

is

$$
{ }^{t} P=\check{T} *: \mathcal{O}^{\prime}(\Omega) \longrightarrow \mathcal{O}^{\prime}(\Omega+K)
$$

with $\widehat{\tilde{T}}(\zeta)=\widehat{T}(-\zeta)$. By the standard argument, it is enough to prove that ${ }^{t} P$ is injective and that the image of ${ }^{t} P$ is weakly closed. In fact, the injectivity of ${ }^{t} P$ shows that the image of $P$ is dense, and the closedness of the image of ${ }^{t} P$ shows the closedness of the image of $P$. Because $T$ is not 0 , the injectivity of ${ }^{t} P$ is clear. Then we will show that the image of ${ }^{t} P$ is weakly closed. To do this, we use the following division lemma, which we can prove in an analoguous way to the proof of Lemma 2.1 in [3].

Lemma 3.2. Let $f, g$ and $h$ be entire functions satisfying $f g=h$, and $K$ and $L$ be two compact convex sets in $\mathbf{C}^{n}$ with $K, L \subset \Omega$. We suppose that for every $\varepsilon>0, f$ and $h$ satisfy the following estimates with constants $A_{\varepsilon}>0$ and $B_{\varepsilon}>0$,

$$
\left\{\begin{array}{l}
\log |f(\zeta)| \leq A_{\varepsilon}+H_{K}(\zeta)+\varepsilon|\zeta| \\
\log |h(\zeta)| \leq B_{\varepsilon}+H_{L}(\zeta)+\varepsilon|\zeta|
\end{array}\right.
$$

for any $\zeta \in \mathbf{C}^{n}$. We also assume that $f$ satisfies the condition $(\mathrm{S})_{\zeta_{0}}$. Then for any $\varepsilon>0$, there exists a compact convex set $M=M_{\varepsilon} \subset \mathbf{C}^{n}$ and $C_{\varepsilon}>0$ such that

$$
\left\{\begin{array}{l}
M \subset \Omega \\
\log |g(\zeta)| \leq C_{\varepsilon}+H_{M}(\zeta) .
\end{array}\right.
$$

End of the proof of the theorem. - Let $\left\{T_{\nu}\right\}$ be a sequence in $\mathcal{O}^{\prime}(\Omega)$ and assume that $\left\{{ }^{t} P T_{\nu}\right\}$ converges to $S \in \mathcal{O}^{\prime}(\Omega+K)$ in $\mathcal{O}^{\prime}(\Omega+K)$. By taking the Fourier-Borel transform, $\widehat{T}(-\zeta) \widehat{T}_{\nu}(\zeta)$ converges to $\widehat{S}(\zeta)$. Then it is well-known that $G(\zeta)=\frac{\widehat{S}(\zeta)}{\widehat{T}(-\zeta)}$ becomes an entire function. By Lemma 3.2 and Theorem 2.1, there exists a compact convex set $M$ and $\mu \in \mathcal{O}^{\prime}\left(\mathbf{C}^{n}\right)$ carried by $M$ such that $\widehat{\mu}(\zeta)=G(\zeta)$ and ${ }^{t} P \mu=\check{T} * \mu=S$, i.e. $S \in \operatorname{Im}^{t} P$. 


\section{THE CHARACTERISTIC SET AND THE CONTINUATION OF HOMO- GENEOUS SOLUTIONS}

Under the condition $(\mathrm{S})_{\zeta_{0}}$, we shall now solve the problem of continuation for the solutions of the homogeneous equation $\left(\mathrm{E}^{\prime}\right)$. For any open set $U \subset \mathbf{C}^{n}$, we set:

$$
\mathcal{N}(U)=\{f \in \mathcal{O}(U) \mid T * f=0\} .
$$

For an open set $V \subset \mathbf{C}^{n}$ with $U \subset V$, the problem is formulated as to get the condition so that the restriction map

$$
r: \mathcal{N}(V) \longrightarrow \mathcal{N}(U)
$$

is surjective.

In order to describe the theorem of continuation, we will prepare the notion of characteristics which is a natural generalization of the case of usual differential operators of finite order with constant coefficients. We define the sphere at infinity $S_{\infty}^{2 n-1}$ by $\left(\mathbf{C}^{n} \backslash\{0\}\right) / \mathbf{R}_{+}$ and consider the compactification with directions $\mathbf{D}^{2 n}=\mathbf{C}^{n} \sqcup S_{\infty}^{2 n-1}$ of $\mathbf{C}^{n}$. For $\zeta \in \mathbf{C}^{n} \backslash\{0\}$, we denote by $\zeta \infty \in S_{\infty}^{2 n-1}$ the equivalence class of $\zeta$, i.e.

$$
\{\zeta \infty\}=\left(\text { the closure of }\{t \zeta \mid t>0\} \text { in } \mathbf{D}^{2 n}\right) \cap S_{\infty}^{2 n-1} \text {. }
$$

For $\varepsilon>0$, we put:

$$
\left\{\begin{array}{l}
V_{\widehat{T}}(\varepsilon)=\left\{\zeta \in \mathbf{C}^{n}|\exp (\varepsilon|\zeta|)| \widehat{T}(\zeta) \mid<1\right\} \\
W_{\widehat{T}}(\varepsilon)=\left(\text { the closure of } V_{\widehat{T}}(\varepsilon) \text { in } \mathbf{D}^{2 n}\right) \cap S_{\infty}^{2 n-1}
\end{array}\right.
$$

Now we define the characteristic set of $T *$.

Definition 4.1. With the above notation, we define the characteristics of $T *$ (at infinity)

$$
\operatorname{Char}_{\infty}(T *)=\text { the closure of } \bigcup_{\varepsilon>0} W_{\widehat{T}}(\varepsilon)
$$

Under the above situation, we can state the theorem of the continuation without proof.

Theorem 4.2. Let $T \in \mathcal{O}^{\prime}\left(\mathbf{C}^{n}\right)$ carried by $K$ and $f \in \mathcal{O}(\Omega+K)$ be a solution of $T * f=0$. Assume that $\widehat{T}(\zeta)$ satisfies the condition $(\mathrm{S})_{\zeta_{0}}$. If $\zeta_{0} \notin \mathrm{Char}_{\infty}(T *)$, then the restriction map

$$
r: \mathcal{N}\left(\mathbf{C}^{n}\right) \longrightarrow \mathcal{N}(\Omega+K)
$$

is surjective, that is, $f$ can be analytically continued to the whole $\mathbf{C}^{n}$. 


\section{References}

[1] V. V. Morzhakov: Convolution equations in convex domains of $\mathrm{C}^{n}$, Compl. Anal. and Appl. '87, Sofia, pp.360-364 (1989).

[2] T. Kawai : On the theory of Fourier hyperfunctions and its applications to partial differential equations with constant coefficients, J. Fac. Sci. Univ. Tokyo, Sect. IA Math., $\underline{\mathbf{1 7}}$, pp.467-517 (1970).

[3] R. Ishimura and Y. Okada: The existence and the continuation of holomorphic solutions for convolution equations in tube domains, Bull. Soc. Math. France, 122, pp.413-433 (1994).

[4] R. Ishimura et J. Okada : Sur la condition (S) de kawai et la propriété de croissance régulière d'une fonction sous-harmonique et d'une fonction entière, Kyushu J. Math. $\underline{48}$, pp.257-263 (1994). 